\title{
Variations in Draining Patterns of Right Pulmonary Veins at the Hilum and an Anatomical Classification
}

\author{
M. S. Rajeshwari and Priya Ranganath \\ Department of Anatomy, Bangalore Medical College \& Research Institute, Bangalore 560 002, India \\ Correspondence should be addressed to M. S. Rajeshwari, rajeshwari.roshan8@gmail.com \\ Received 28 February 2012; Accepted 9 May 2012 \\ Academic Editors: B. O. Ibe and S. D. Qanadli
}

Copyright ( $) 2012$ M. S. Rajeshwari and P. Ranganath. This is an open access article distributed under the Creative Commons Attribution License, which permits unrestricted use, distribution, and reproduction in any medium, provided the original work is properly cited.

\begin{abstract}
Pulmonary veins carry oxygenated blood from the lungs to the left atrium. Variations are quite common in the pattern of drainage. The present study was undertaken to evaluate the incidence of different draining patterns of the right pulmonary veins at the hilum by dissecting the human fixed cadaveric lungs. Clinically, pulmonary veins have been demonstrated to often play an important role in generating atrial fibrillation. Hence, it is important to look into the anatomy of the veins during MR imaging and CT angiography. In $53.8 \%$ of cases, the right superior lobar vein and right middle lobar vein were found to be united together to form the right superior pulmonary vein. In contrast to this, in $11.53 \%$ of cases, right middle lobar vein united with the right inferior lobar vein to form the right inferior pulmonary vein, while in $26.9 \%$ of cases, the right superior lobar vein, right middle lobar vein, and right inferior lobar vein drained separately.
\end{abstract}

\section{Introduction}

Pulmonary veins drain oxygenated blood from the lungs to the left atrium of the heart. The lobular tributaries lie mainly in the interlobular septa. One main vein drains each bronchopulmonary segment usually on the anterior surface of the corresponding bronchus. Veins also run between the segments and on the mediastinal and fissural surfaces of the lungs; some veins cross the line of fissure when the fissures are incomplete. The veins of the right medial segment of inferior lobe frequently arise from the veins of the middle lobe. The superior right pulmonary vein drains the right superior and middle lobe and thus corresponds to the superior left pulmonary vein which drains the left superior lobe. The right inferior pulmonary vein and left inferior pulmonary veins drain the corresponding inferior lobes [1]. The present study is to ascertain the incidence of different draining patterns of the right pulmonary vein at the hilum as it is clinically very important to look into the anatomy of the veins during $\mathrm{MR}$ imaging and CT angiography.

\section{Material and Methods}

The study involved twenty-six formalin-fixed adult cadaveric lungs of unknown sex from Bangalore Medical College \& Research Institute, Bangalore, Karnataka. The dissection initially involved identifying the structures at the hilum. The pulmonary veins were picked up and their tributaries were traced peripherally by meticulously dissecting the lung tissue using blunt forceps. Interestingly different patterns in the drainage of pulmonary veins were noted.

\section{Results}

The different draining patterns noted are as follows. In 53.8\% of dissected lungs the right superior lobar vein and right middle lobar vein united together to form the right superior pulmonary vein. The right superior lobar vein drained the apical and anterior segment of right upper lobe and also the medial and lateral segments of the right middle lobe from the anterior surface, whereas veins from the posterior segment of 


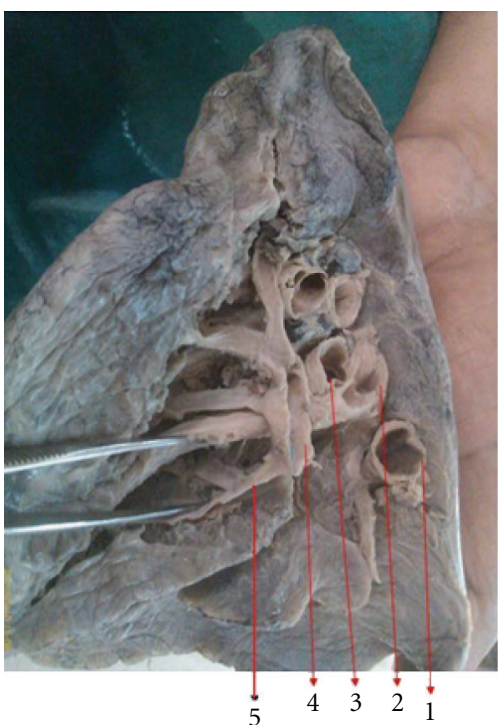
(1) IPV
(2) Bronchus
(4) SPV (SLV + MLV)
(3) Artery
(5) Middle lobar vein forming trunk before draining into SPV

FIGURE 1
Classification into four types depending on the incidence:

Type A:

RSLV and RMLV join to form RSPV (53.8\%).
RILV forms RIPV.

Type A1: RMLVs form a common trunk and drain into RSLV.

Type A2: RMLVs remain separate and drain into RSLV.

Type B:

RSLV, RMLV, and RILV drain separately (26.9\%).

Type-C:

RSLV forms RSPV.

RMLV and RILV join to form RIPV (11.53\%).

Type D: RSLV, RMLV, and RILV join to form RUSCPV (3.8\%).

\section{Discussion}

Different types of drainage patterns of the pulmonary veins of the right lung have been reported in the literature by the radiologists. In our study, a classification is given concentrating on all the three lobar veins of the right lung.

Variation in the number and the course of pulmonary veins is not uncommon and till now was the subject of only case reports. Although a classification is given by $[2,3]$, the present classification is given by considering the structures at the level of hilum.

The frequency of atrial arrhythmias is high in patients with separate ostium for the right middle lobar vein than those with other patterns [3]. If more than one pulmonary vein drains anomalously, the volume is usually sufficient to produce the characteristic pattern of the right ventricular diastolic overload.

The ectopic beats arise from these anomalous veins. This greater variability in pulmonary venous anatomy than expected could substantially alter the success rate of radiofrequency ablation, as ectopic foci may go untreated in variant veins [3]. Pulmonary veins play a critical role in the pathophysiology of atrial fibrillation. Knowledge of normal pulmonary venous anatomy is essential for preablation planning and for evaluation of postablation complication [4].

The pulmonary veins are the dominant source of ectopic depolarization initiating atrial fibrillation in the majority of patients with paroxysmal atrial fibrillation and catheter ablation has emerged, as realistic treatment strategy targeting these pulmonary venous triggers [5]. The anatomic variation of middle lobar vein draining into the inferior pulmonary vein is surgically important because when performing the right lobar lobectomy, the division of RIPV may lead to inferior lobar vein, and all the three right lobar veins drained separately (Figure 6). 


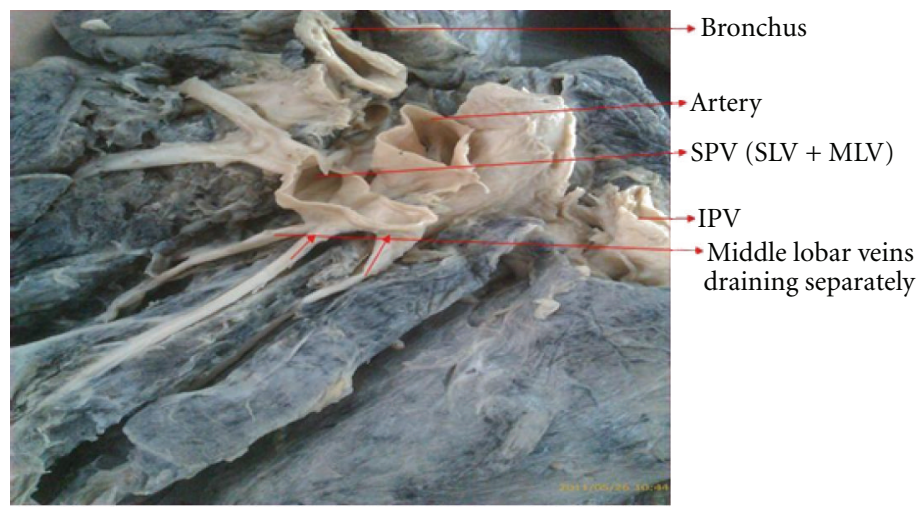

Figure 2

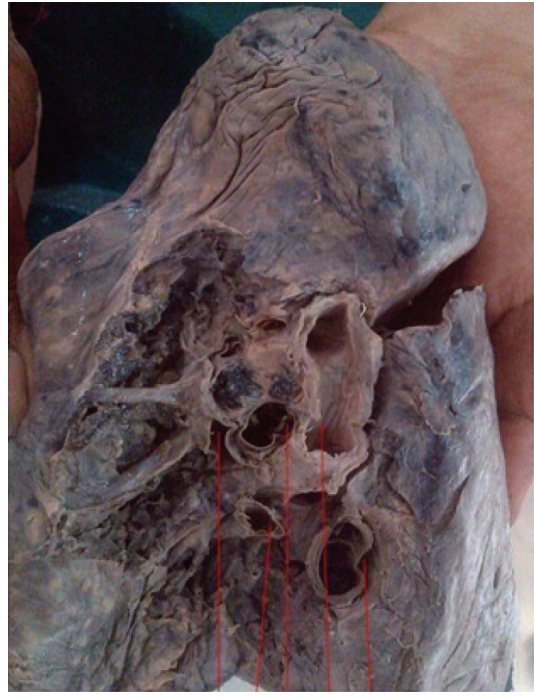

$\begin{array}{lllll}5 & 4 & 3 & 2 & 1\end{array}$
(1) ILV
(2) Bronchus
(4) MLV
(3) Artery
(5) SLV

Figure 3

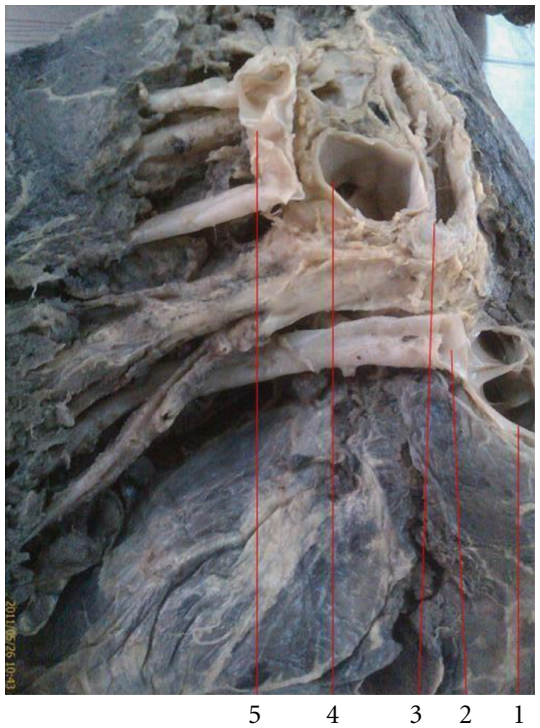
(1) ILV
(4) Artery
(2) MLV
(5) SLV
(3) Bronchus

Figure 4 severe hemorrhage or improper ligation of drainage veins result in oedema, which can be life threatening if the surgeon overlooks the anomalies, which can lead to an increase in the surgical morbidity [6].

In the present study, in one case, the SLV was present posterior to the bronchus, which is not as infrequent as what was previously believed [7]. Preoperative identification of this variation is useful for decreasing the incidence of unexpected intraoperative bleeding [7]. Electrophysiologic studies [8] reported that five atrial fibrillation foci arose from RMPV. The coupling interval between the ectopic beat of the atrial fibrillation and the sinus beat was longer in RMPV than RSPV. Ectopy from RMPV can initiate atrial fibrillation. Knowledge of the anatomical positions and the morphology of the pulmonary veins by magnetic resonance angiography is useful for electrophysiologist as it facilitates catheter positioning during pulmonary vein ablation [9].

\section{Conclusion}

In this study, variations in pulmonary venous anatomy were seen in $46.03 \%$ of dissected lungs. These variations helped us to come to a conclusion on an anatomical classification, depending on the drainage patterns of the right pulmonary vein at the hilum.

The results confirm that there is considerable variation in the anatomy of pulmonary veins.

With the increasing use of cardiovascular imaging, variations in the pulmonary vein anatomy have become more appreciated, hence detailed knowledge of pulmonary venous 


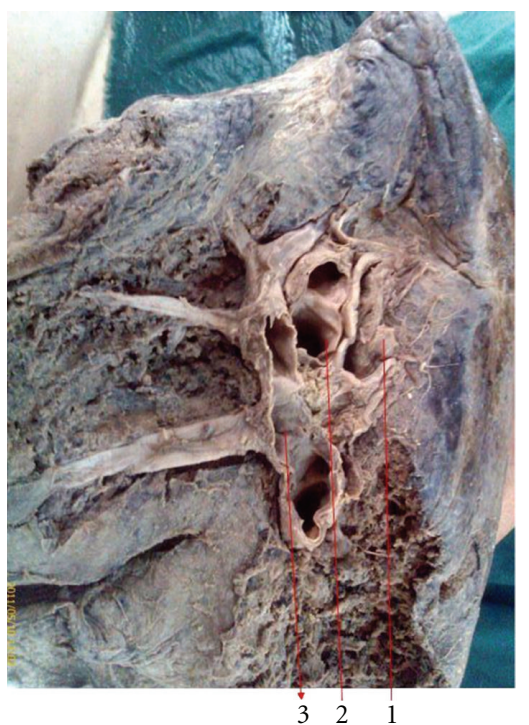

(1) Bronchus

(2) Artery

(3) SUCPV

Figure 5


(1) ILV
(4) SLV
(2) MLV
(5) Artery
(3) Bronchus

Figure 6

anatomy and drainage pattern is important during mapping and ablation procedures.

\section{Key Message}

Pulmonary veins play a pivotal role in atrial fibrillation. Anatomical variations of these pulmonary veins are arrhythmogenic potential. Variations in the pulmonary veins are not rare but need a detailed study before performing any lung surgeries. CT angiography and MR imaging can pick up such variations to avoid unwanted complications on the table.

\author{
Abbreviations \\ RSLV: Right superior lobar vein \\ RMLV: Right middle lobar vein \\ RSPV: Right superior pulmonary vein \\ RILV: Right inferior lobar vein \\ RIPV: Right inferior pulmonary vein \\ RUSCPV: Right unilateral single central pulmonary vein.
}

\section{Acknowledgments}

The authors are grateful to Dr. K. S. Jayanthi and Mr. Sameer for their support.

\section{References}

[1] G. J. Romanes, Blood Vessels of Lungs, Cunningham's Manual of Practical Anatomy, Oxford University, London, UK, 14th edition, 1966.

[2] F. Yazar, O. Ozdogmus, E. Tuccar, A. Bayramoglu, and H. Ozan, "Drainage patterns of middle lobe vein of right lung: an anatomical study," European Journal of Cardio-Thoracic Surgery, vol. 22, no. 5, pp. 717-720, 2002.

[3] E. Marom, J. Herndon, Y. Kim, and H. P. McAdams, "Variations in pulmonary venous drainage to the left atrium: implications for radiofrequency ablation," Radiology, vol. 230, no. 3, pp. 824$829,2004$.

[4] T. H. Hauser, D. C. Peters, J. V. Wylie, and W. J. Manning, "Evaluating the left atrium by magnetic resonance imaging," EP Eurospace, vol. 10, supplement 3, pp. iii22-iii27, 2008.

[5] C. Thorning, M. Hamady, J. V. P. Liaw et al., "CT evaluation of pulmonary venous anatomy variation in patients undergoing catheter ablation for atrial fibrillation," Clinical Imaging, vol. 35, no. 1, pp. 1-9, 2011.

[6] S. Sugimoto, O. Izumiyama, A. Yamashita, M. Baba, and T. Hasegawa, "Anatomy of inferior pulmonary vein should be clarified in lower lobectomy," The Annals of Thoracic Surgery, vol. 66, no. 5, pp. 1799-1800, 1998.

[7] K. Asai, N. Urabe, K. Yajima, K. Suzuki, and T. Kazui, "Right upper lobe venous drainage posterior to the bronchus intermedius: preoperative identification by computed tomography," The Annals of Thoracic Surgery, vol. 79, no. 6, pp. 1866-1871, 2005.

[8] H. M. Tsao, M. H. Wu, W. C. Yu et al., "Role of right middle pulmonary vein in patients with paroxysmal atrial fibrillation," Journal of Cardiovascular Electrophysiology, vol. 12, no. 12, pp. 1353-1357, 2001.

[9] F. Carreras, E. Guillaumet, S. Pujadas et al., "Morphologic study of the pulmonary veins by magnetic resonance angiography," Revista Espanola de Cardiologia, vol. 58, no. 10, pp. 1226-1229, 2005. 


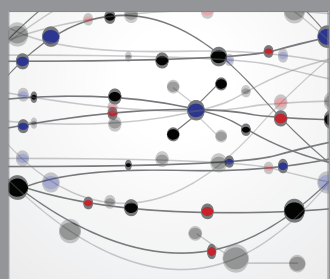

The Scientific World Journal


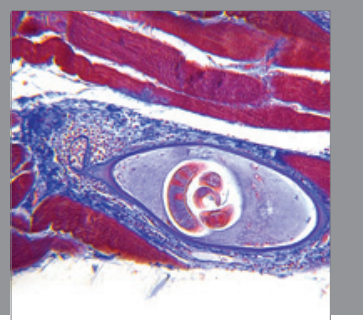

Gastroenterology

Research and Practice
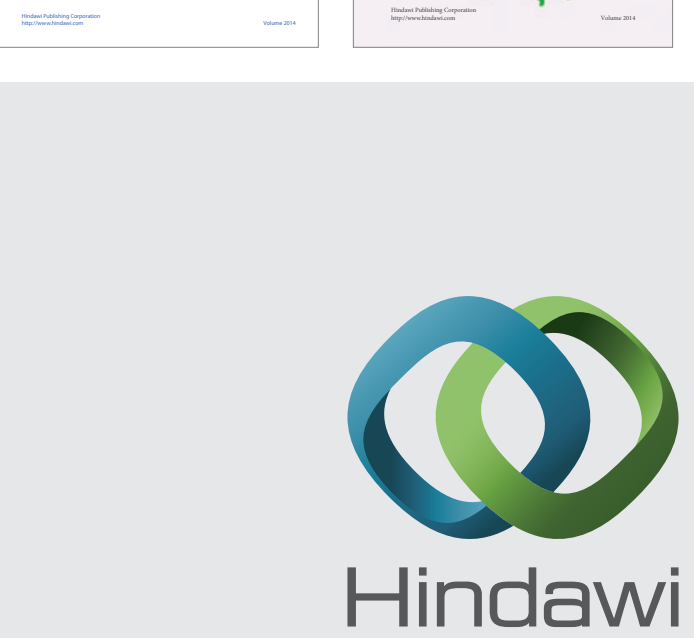

Submit your manuscripts at

http://www.hindawi.com
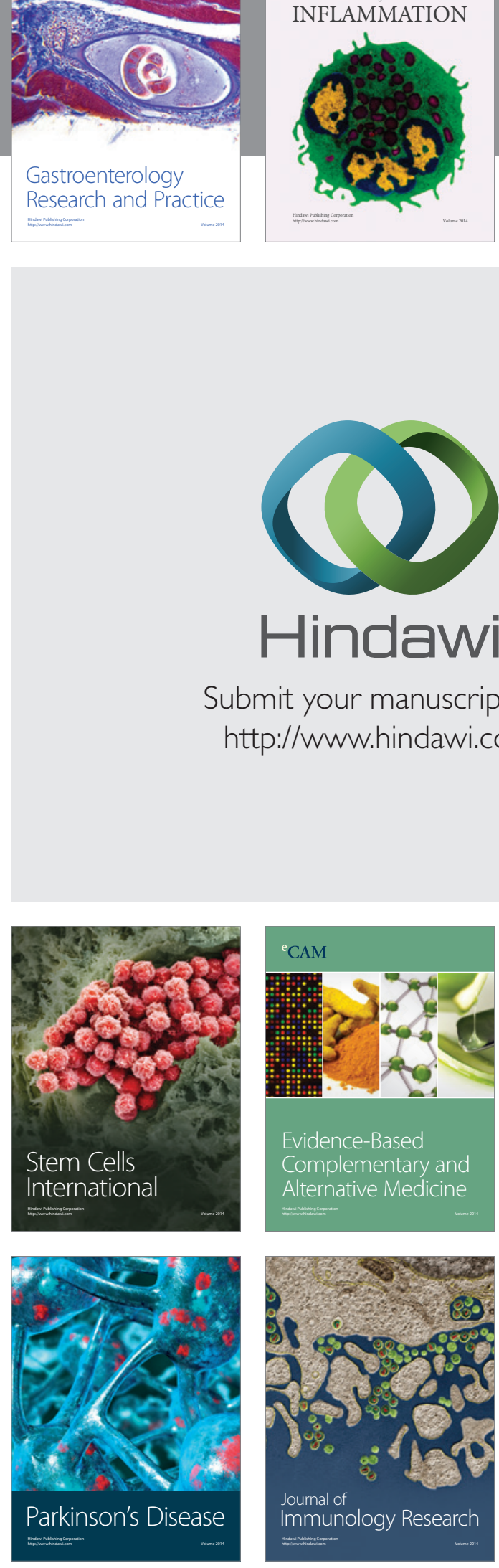

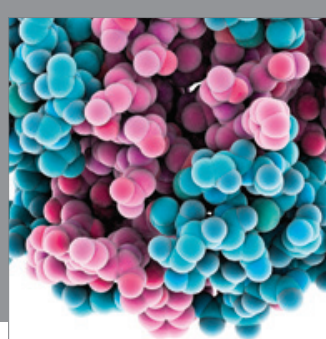

Diabetes Research
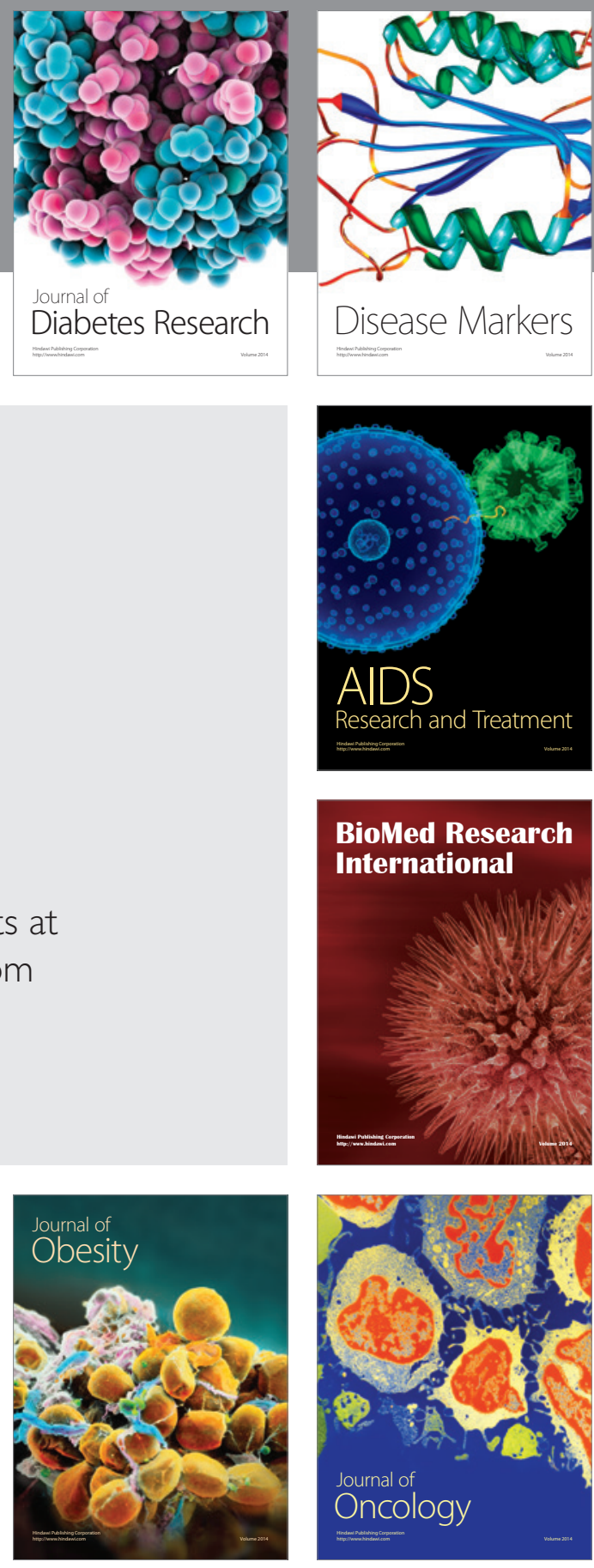

Disease Markers

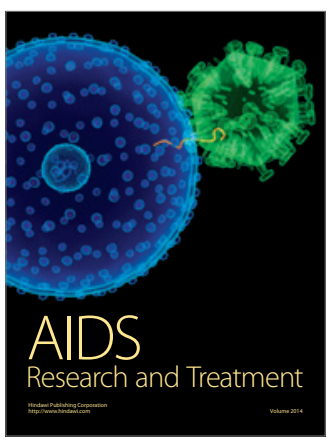

BioMed Research

International
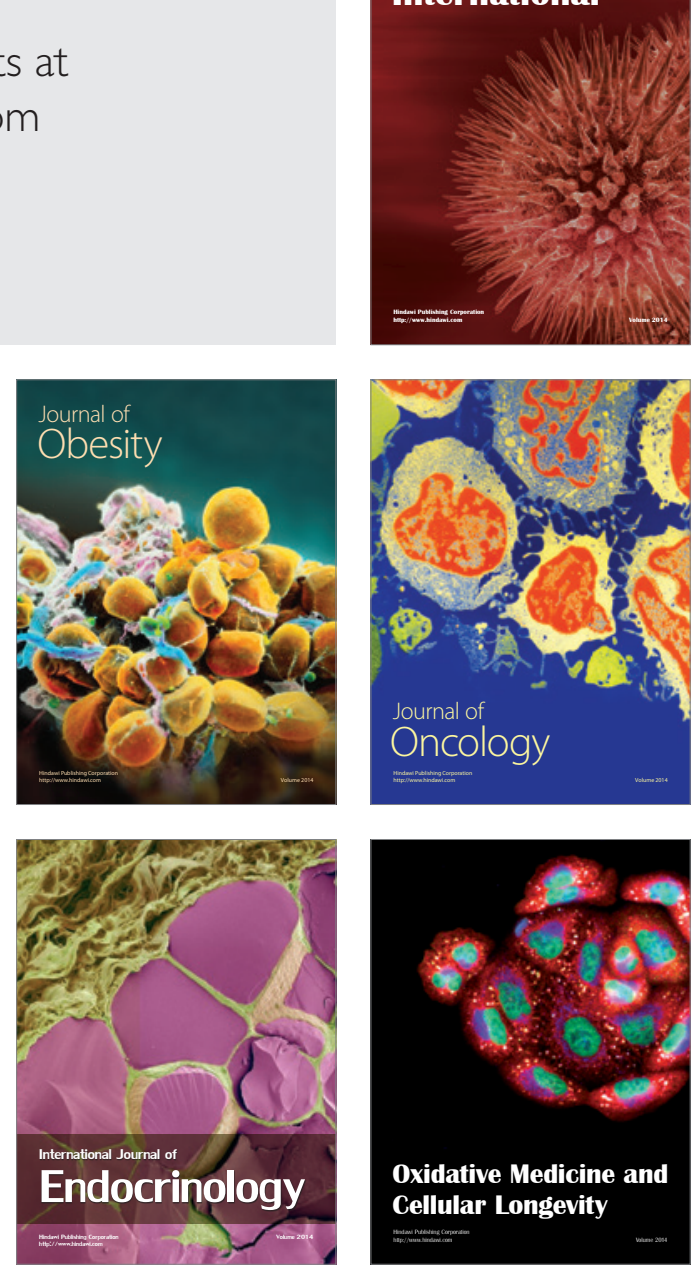